\title{
Konzepte und Maßnahmen zum Umgang mit soziotechnischen Herausforderungen bei der Entsorgung radioaktiver Abfälle
}

\section{Ein einleitender Beitrag}

Achim Brunnengräber, Ana María Isidoro Losada, Bettina Brohmann, Peter Hocke

Die Endlagerung von Abfällen - insbesondere von hochradioaktiven - stellt eine technische sowie gesellschaftspolitische Herausforderung dar - und sie steht noch am Anfang. Trotz jahrzehntelanger Nutzung der Atomenergie wurde die möglichst sichere Entsorgung der Abfälle nicht mit der erforderlichen Dringlichkeit behandelt oder wurde sogar verschleppt. Wie schwierig der Weg dorthin ist, zeigte sich an den konkreten Endlagerprojekten, die kaum positive Orientierungen geben können. Weder zeigten große bundesdeutsche Energieversorgungsunternehmen (EVU) als Betreiber der Atomkraftwerke (AKW) großes Interesse, die Konflikte um den Standort Gorleben zu entschärfen, noch gelang es der Politik, die Auseinandersetzungen insbesondere in Niedersachsen um das geplante Endlager Schacht Konrad sowie die marode Schachtanlage Asse II nachhaltig konstruktiv zu wenden. Über Jahrzehnte hinweg ist es nicht gelungen, eine technische sowie eine politische Lösung für die Abfälle zu finden, die bis zur Reaktorkatastrophe in Fukushima in deutschen Atomkraftwerken fortlaufend produziert wurden und bis zum abgeschlossenen Atomausstieg 2022 weiterhin produziert werden (Hocke/Kallenbach-Herbert 2015).

Mit einer Mischung aus Entscheidungsblockaden und der Strategie des Abwartens ist die Einlagerung hochradioaktiver Abfälle in die zweite Hälfte dieses Jahrhunderts verschoben worden. Dies führt dazu, dass nicht nur die gegenwärtige Bevölkerung, sondern auch die nachfolgenden Generationen in ein "Jahrhundertprojekt « (Brunnengräber 2017) eingebunden werden. Erst nach der Reaktorkatastrophe im AKW Fukushima Daiichi veränderten sich die politischen Rahmenbedingungen. Mit dem Atomausstieg, der in Deutschland bis Ende 2022 vollzogen sein soll, dem Standortauswahlgesetzt (StandAG 2013, geändert in 2017) und der Arbeit der Endlagerkommission (20142016) sowie der darauffolgenden Neuausrichtung der Entsorgung der hochradioaktiven Abfälle ist bis zu einem gewissen Grad eine neue und konstruktivere Zusammenarbeit zwischen den zentralen Akteuren der Endlagerpolitik entstanden (Grunwald 2016, 
Kamlage et al. 2019). Diese sehen sich allerdings mit erheblichen und ineinandergreifenden Problemen konfrontiert, wie sich auch nach der Veröffentlichung des Zwischenberichts Teilgebiete der Bundesgesellschaft für Endlagerung (BGE) im September 2020 zeigte (BGE 2020). Nach den Vorschlägen, welche die BGE als Vorhabenträger in diesem Bericht unterbreitet hat, kommt knapp die Hälfte der Fläche der Bundesregierung als Standort für ein Endlager in Frage. Mit diesem Bericht ist die erste Phase der Standortsuche vorangetrieben. In den nächsten beiden Phasen werden Standorte für die übertägige und darauffolgend für die untertägige Erkundung ermittelt.

Die hochradioaktiven Abfälle befinden sich derweil in Behältern in zentralen Zwischenlagern oder in Zwischenlagern neben den AKW-Standorten, die über ganz Deutschland verteilt sind. Laut Bundesamt für die Sicherheit der nuklearen Entsorgung (BASE), der Aufsichtsbehörde des Verfahrens, bieten sie »aktuell den notwendigen Schutz für Mensch und Umwelt«. Sie seien aber nur eine Übergangslösung, weil »Stacheldraht und Wachmannschaften nicht den gleichen Schutz gewährleisten, wie ihn ein Lager in stabilen Gesteinsschichten tief unter der Erde bietet « (BASE 2020: 3). Zugleich ist die Zwischenlagerung der atomaren Hinterlassenschaften auf den langen Zeitstrahl, der sich über die Suche nach einem Standort, den Bau einer Lagerstätte, die Einlagerung der dafür geeigneten Behälter und die Nachbetriebsphase erstreckt, nicht ausgelegt. Die Zwischenlagerung der abgebrannten Brennelemente wird länger als die 40 Jahre dauern, für die die Zwischenlager genehmigt sind. Alle bisherigen Zwischenlager-Genehmigungen laufen zwischen 2034 und 2047 aus. Auch daraus leitet sich die Dringlichkeit der Standortsuche ab.

Dem Verfahrensprinzip von staatlichen Entscheidungen, der Verkündung dieser und ihrer Verteidigung, kann dabei nicht mehr, wie in den frühen Jahren des atomaren Energiezeitalters, gefolgt werden. Eine Öffentlichkeitbeteiligung ist darin nicht vorgesehen, wird durch das StandAG nun aber sogar gesetzlich vorgeschrieben. Bei der Entsorgung greifen technische, politische, soziale und ökonomische Prozesse auf komplexe Weise ineinander. Sicherheit und Zuverlässigkeit werden nicht allein - so auch die Ausgangsüberlegung für diesen Band - durch den sicheren Einschluss oder die möglichst beste Technologie gewährleistet. Beides entwickelt sich vielmehr innerhalb von soziotechnischen Systemen, in denen natur- und ingenieurwissenschaftliche Lösungsansätze sowie soziale und politische Erwartungen ineinandergreifen (Kallenbach et al. 2018, Hocke 2016). Das dabei verfolgte Ziel, das auch im StandAG festgelegt wurde, lautet, den langzeitsicheren Einschluss der hochradioaktiven Abfälle für einen Zeitraum von einer Million Jahre anzustreben.

Die Neuorganisation des Auswahlverfahrens für einen Endlagerstandort durch das StandAG sieht nun ein partizipatives, wissenschaftsbasiertes, transparentes, selbsthinterfragendes und lernendes Verfahren vor (\$1 Zweck des Gesetzes, StandAG 2017). Der lange verfolgte Entsorgungspfad, der sich auf das Erkundungsbergwerk Gorleben konzentrierte, wurde mit dem Zwischenbericht Teilgebiete auf wissenschaftsbasierter Grundlage beendet (BGE 2020). Gorleben wurde darin als nicht geeigneter Standort ausgewiesen. Damit wurde auch ein jahrzehntelanger gesellschaftlicher Konflikt und lock in abgeschlossen. Zugleich wurde mit dieser Entscheidung ein Entwicklungspfad eröffnet, der nicht nur durch neue Institutionen, einen neuen Rechtsrahmen und neue Verfahrensschritte geprägt ist; es ist auch ein Versuch des Ausbalancierens zwischen 
gesellschaftlichen Erwartungen und technischen Lösungsansätzen. Die wesentlichen soziotechnischen Schwierigkeiten im Umgang mit hochradioaktiven Abfällen lösen sich dadurch aber längst nicht auf. Die Entsorgung umfasst auch weiterhin komplexe Abläu$\mathrm{fe}$, in denen politische, ingenieurstechnische und rechtliche Dimensionen ineinandergreifen. Die sozialen Abläufe werden wiederum getragen von einer Vielzahl beteiligter Akteure mit unterschiedlichen Interessen, Verantwortlichkeiten und Erwartungen.

Zentrale Aspekte dieser komplexen Zusammenhänge beleuchten wir in dem hier vorgelegten Sammelband aus politik- und sozialwissenschaftlicher Perspektive sowie aus der Perspektive der Technikfolgenabschätzung. Die engen Interdependenzen zwischen technischen Prozessen und sozialen Dynamiken sind in der Entsorgungsforschung bisher nicht genügend betrachtet worden. Es fehlt sowohl an wissenschaftlichen Konzepten, mit denen sich die Wechselwirkungen besser erfassen lassen, als auch an wissenschaftlichen Analysen, die diese Interdependenzen beleuchten. Aus diesem doppelten Defizit leiteten sich die Fragestellungen ab, denen in den Beiträgen in diesem Sammelband nachgegangen wird. Es wird aufgezeigt, wie sich die soziotechnischen Wechselwirkungen und damit zusammenhängenden Herausforderungen bei der Endlagerung analysieren lassen und welche Ergebnisse auf diese Weise erzielt werden können. Darüber hinaus ist es das Ziel in diesem Band, Systemwissen (über die Standortsuche), Orientierungswissen (über die Verfahrensschritte) und Zielwissen (zur Verbesserung der Standortsuche) zu vermitteln.

\section{Zum Aufbau des Bandes}

\section{Teil I: Konzeptionelle und geschichtliche Einblicke}

Im ersten Teil des Sammelbandes findet eine Annäherung an soziotechnische Dimensionen statt, die für die Endlagerung von Relevanz sind. Des Weiteren wird eine historische Verortung der Entsorgung geliefert. Die Suche nach Konzepten für ein soziotechnisches Verständnis der Entsorgung wird im Beitrag "Welche Unterscheidungen braucht die Endlagerforschung?« von Andreas Lösch eröffnet, der sich mit grundlegenden Konzepten aus der interdisziplinären Technik- und Innovationsforschung auseinandersetzt. Für ihn stellt die nukleare Entsorgung einen besonderen Konflikt dar, der sich von anderen Fällen der Forschung zu Technikgestaltung unterscheidet, da Sachzwänge und geologische Gegebenheiten das Errichten eines Tiefenlagers für hochradioaktive Abfälle besonders prägen. Gleichzeitig zeigen Technikfolgenabschätzung (TA) und Science and Technologies Studies (STS), dass die Ko-Produktion von Technik und Sozialem in modernen demokratischen Gesellschaften immer möglich ist, wenn bestimmte Voraussetzungen erfüllt sind. Der Beitrag weist unter anderem auf die besondere Rolle von »System-Buildern« hin, denen es in der Vergangenheit immer wieder gelang, radikale Innovationen durchzusetzen. Weiterhin wird in diesem Beitrag in die soziologische Grundfigur der sozialen Schließung eingeführt und die wechselseitige Bedingtheit gesamtgesellschaftlicher Dynamiken sowie das kollektive Handeln in soziotechnischen Regimen und innovationsfreudigen Nischen thematisiert. 
In den beiden darauffolgenden Beiträgen werden soziotechnische Dimensionen der Endlagerung aus historischer Perspektive betrachtet. Lutz Mez und Daniel Häfner gehen in ihrem Beitrag »Nukleare Technopolitik in der BRD« auf die Irreversibilität ein, die durch das Atomzeitalter geschaffen wurden. Die unterschiedlichen Interessenlagen waren nur in einem "starken Atomstaat « umzusetzen; sobald Mitsprache beispielsweise durch Umweltverträglichkeitsprüfungen ermöglicht wurde, waren viele der nuklearen Projekte nicht mehr durchzuführen. Der »starke Atomstaat« muss nun, am Ende der Nutzung der Kernenergie, durch Partizipationsangebote sehr »weich « sein, um ein Endlagerprojekt umsetzen zu können. In dem Beitrag "Vom starken zum weichen Atomstaat« von Achim Brunnengräber wird das Buch »Der Atom-Staat« von Robert Jungk (1977) einer neuerlichen Lektüre unterzogen um zu verdeutlichen, dass in den Anfangsjahren des Nuklearzeitalters aus politisch-strategischen Gründen das Soziale vom Technischen getrennt wurde. Zugleich waren die Schnittstellen schon damals offensichtlich, sie hätten aber nur dann verknüpft werden können, wenn die Atompolitik demokratisch organisiert worden wäre. Eine Durchsetzung mittels der starken Hand des Staates wie bei der Atomkraft kann bei der Endlagerung aber nicht gelingen. So lässt sich aus der Lektüre vielmehr folgern, dass heute der »weiche Endlagerstaat« das Problem im Dialog mit der Gesellschaft lösen muss.

Nach dieser konzeptionellen Vorarbeit und historischen Verortung werden in den folgenden Beiträgen erste empirisch fundierte Zugänge zu den soziotechnischen Herausforderungen der Entsorgung vorgestellt. Im Rahmen einer empirischen Erhebung mittels semistrukturierter Leitfadeninterviews wurden von Achim Brunnengräber, Ana María Isidoro Losada, Rosaria Di Nucci und Dörte Themann qualitative Daten zum Verständnis von soziotechnischen Aspekten der Standortsuche und der Entsorgung von hochradioaktiven Abfällen ausgewertet. Im Ergebnis zeigt sich, dass der Begriff des Soziotechnischen sowie der Zusammenhang der sozialen und technischen Dimensionen der Endlagerung für die meisten Interviewten zunächst schwer zu fassen ist. Gleichzeitig lässt sich allerdings beobachten, dass die Wechselwirkungen implizit von Bedeutung sind und die soziotechnischen Aspekte der Standortsuche und der Endlagerpolitik, sobald sie benannt werden, auch als politisch relevant angesehen werden. Sie dienen vielmehr der Bewertung der Entsorgungssituation in Deutschland, wenn etwa die Frage der technischen Machbarkeit der Rückholung der Abfälle aus dem Endlager und das gesellschaftliche Bedürfnis nach möglichst großer Sicherheit durch den Verschluss der Anlage verhandelt werden (»Der Atommüll - eine soziotechnische Tatsache«).

Eine andere Herangehensweise wird von Dörte Themann und Achim Brunnengräber mit der Identifizierung von Analoga gewählt, um die soziotechnischen Dynamiken der Endlagerung abschätzen zu können. Anhand eines Kriterienkatalogs, der verschiedene Aspekte und Ebenen soziotechnischer Ensembles abdeckt, wurden Fracking, Windenergie und Carbon Dioxide Capture and Storage (CCS) vergleichend betrachtet und die Erkenntnisse auf das Ensemble der Endlagerung radioaktiver Abfälle übertragen. Es wird festgestellt, dass die Dynamiken und Prozesse aus anderen Großinfrastrukturprojekten durchaus einen indirekten Erfahrungshorizont darstellen und produktiv für die Analyse der soziotechnischen Prozesse der Standortsuche und Entsorgung genutzt werden können, etwa was den Umfang und den Zeitpunkt der Öffentlich- 
keitsbeteiligung anbelangt. Auch stellt die kritische Öffentlichkeit einen neuen Schlüsselakteur in den soziotechnischen Ensembles dar. Denn die Themen Sicherheit und Risiko der Infrastrukturprojekte deuten noch auf erhebliche Unsicherheiten hin, die vor allem einen offenen Wertedialog und die Integration verschiedener Wissenstypen erfordern. Der Ansatz der soziotechnischen Analoga könnte in zukünftigen Forschungsvorhaben genutzt werden, um die hier formulierten Kriterien zu erweitern und die systemischen Wechselwirkungen zwischen diesen sowie zwischen Akteursgruppen noch vertiefender zu analysieren („Soziotechnische Analoga als Erfahrungshintergrund für ein Endlager«).

\section{Teil II: Regulierung und Interdependenzen}

Aus soziotechnischer Perspektive gibt es nicht einen einzelnen Entsorgungspfad, sondern vielmehr verschiedene. Der bestehende Pfad wird allerdings durch Institutionen, Abhängigkeiten und politische Entscheidungsprozesse so gefestigt, dass eine Abkehr von den einmal eingeschlagenen Verfahrensschritten nur unter Einsatz von Wissen, gesellschaftlichem Engagement oder technischen Innovationen möglich ist. Mehr oder weniger tiefgreifende Neuregulierungen sind über lange Zeiträume hinweg jedoch wahrscheinlicher als die Beibehaltung des status quo. Vor diesem Hintergrund stellt das Konzept der Pfadabhängigkeiten einen fruchtbaren Ansatz für die Analyse der Entsorgungspolitik von hochradioaktiven Abfällen in der Bundesrepublik Deutschland dar. Zwar ist die Geschichte des Umgangs mit Atommüll durch spezifische (inter-)nationale Pfadabhängigkeiten geprägt, dennoch deuten verschiedene Ereignisse und Prozesse in den vergangenen Jahrzehnten auf kontextbedingte Pfadbrechungen und nationale Besonderheiten hin.

Von Ana María Isidoro Losada wird in dem Beitrag "Pfadabhängigkeiten in der Endlagerpolitik« der Frage nachgegangen, inwieweit der Umgang mit den hochradioaktiven Abfällen sowie das gegenwärtige Standortauswahlverfahren nach wie vor durch Pfadabhängigkeiten geprägt sind. Dafür wird zunächst geklärt, inwieweit die Genese des gegenwärtigen Endlagerkonzeptes durch sich (international) verändernde technische Grundannahmen, aber auch politisch-ökonomische Schwerpunktsetzungen bzw. -verlagerungen in der Vergangenheit mitgeprägt wurde. Es wird gezeigt, dass auch die jeweiligen gesellschaftspolitischen Auseinandersetzungen und Erwartungen im Umgang mit hochradioaktiven Abfällen die jeweilige Endlagerpolitik beeinflussten. Abschließend werden Voraussetzungen abgeleitet, die erfüllt sein müssen, um den künftigen Pfad für das Standortauswahlverfahren, aber auch die Entsorgung insgesamt politisch gestalten zu können.

Daraus leitet sich die Frage ab, wie es zu diesen Veränderungen in der Endlagerpolitik in Deutschland kam. Mit Blick auf das aktuelle Standortauswahlverfahren stellt sich angesichts der diversen Problemfelder darüber hinaus die Frage, wie politische Entscheidungsträger*innen oder zivilgesellschaftliche Akteure mit der Komplexität, den Unsicherheiten und Ambivalenzen im Bereich der Endlagerung umgehen und wie diese in politischen Entscheidungsprozessen abgebildet werden. Hierzu wird in vier Beiträgen mit je unterschiedlichen Forschungsobjekten das Science-Policy Interface - also das spezifische Verhältnis zwischen Wissenschaft, Politik und Gesellschaft - der Zwischen- 
und Endlagerung betrachtet. Dies geschieht im speziellen am Beispiel von Expert*innendissensen, die in Kommissionen, Arenen, Lernräumen und Diskursen verortet werden.

Im Beitrag von Ana María Isidoro Losada, Dörte Themann und Maria Rosaria Di Nucci wird eine vergleichende Analyse verschiedener Beratungsgremien und -kommissionen vorgestellt, die die Politik im Laufe der bundesdeutschen Geschichte zur Endlagerung seit 1980 beraten haben bzw. immer noch beraten. In diesem Zusammenhang werden die Enquete-Kommission Zukünftige Kernenergiepolitik (1979-1983), der Arbeitskreis Endlagerung (AkEnd) (1999-2002), die Entsorgungskommission (ESK) (2008-heute), die Endlagerkommission (EndKo) (2014-2016) und das Nationale Begleitgremium (NBG) (2016 bis heute) anhand von vier Kriterien analysiert: (1) Pluralität in der (disziplinären) Zusammensetzung, (2) Transparenz von Beratung und Entscheidungsfindungsprozessen, (3) Einfluss auf Entscheidungen und Policy sowie (4) Distanz bzw. Unabhängigkeit zwischen Beratenden und zu Beratenden. Hierbei zeigt sich im Zeitverlauf ein Wandel, der sich vor allem in der Zusammensetzung der verschiedenen Beratungsgremien sowie der stetig wachsenden Relevanz des Aspekts einer aktiven und befähigenden Öffentlichkeitsbeteiligung in den Empfehlungen zum Standortauswahlverfahren widerspiegelt. Das NBG stellt hierbei eine aussichtsvolle Annäherung an das dar, was Gibbons (1999) socially robust knowledge nannte (»Rolle und Entwicklung politischer Beratungs- und Begleitgremien nach dem Konzept des Science-Policy Interfaces «).

Nach diesem Überblick über zeitliche Wandlungsprozesse im Science-Policy Interface wird von Ana María Isidoro Losada, Dörte Themann und Daniel Häfner der Blick auf aktuelle Dissense in der Endlagerdebatte gerichtet. Widerstreitende Aussagen von Expert*innen prägten über Jahrzehnte nicht nur die Debatten über die zivile Nutzung der Atomenergie in der Bundesrepublik insgesamt, sie prägten auch auf besondere Weise die jahrelangen Auseinandersetzungen um den Standort Gorleben als Endlager für hochradioaktive Abfälle. Um die Bedeutung und Auswirkungen von aktuellen Expert*innendissensen im Rahmen des neu eröffneten Standortauswahlverfahrens bewerten zu können, haben die Autor*innen ein Konzept aus vier verschiedenen Arenentypen entwickelt und Interviews mit verschiedenen Stakeholdern geführt. Mithilfe dieses Konzeptes konnte, am Beispiel der Auseinandersetzung um glaziale Ereignisse als potenzielles Ausschlusskriterium für einen Endlagerstandort, Schlussfolgerungen gezogen werden. So fehlen diskursive Räume, die einen tiefgehenden Austausch zwischen den staatlichen Institutionen und unabhängig forschenden Einrichtungen sicherstellen. Bei den zivilgesellschaftlichen und wissenschaftlichen Interviewpartner*innen besteht zudem der Eindruck, dass Dissense zwischen den Institutionen ge- und vermieden werden, was sich belastend auf das Vertrauen in das Verfahren auswirken kann. Die Ergebnisse deuten weiterhin auf eine hohe Intransparenz im Wechselspiel von politischer Steuerung und Expert*innenwissen hin. Bei einigen Arenen zeigt sich zwar eine Öffnung des diskursiven Raumes zu strittigen Fragen der Endlagersuche, doch zeichnet sich gerade bei den staatlichen Institutionen noch keine konkrete Strategie ab, wie ein produktives Verhältnis zur Gegenexpertise transparent und öffentlichkeitswirksam abgesichert werden kann (»Arenen zur Austragung von Dissensen in der Endlagerpolitik«). 
Um wesentliche Faktoren und Lernräume aufzuzeigen, die beim produktiven Aufgreifen von Expert*innendissensen für politische Entscheidungsprozesse zum Tragen kommen, greift der nächste Beitrag zu diesem Themenkomplex von Dörte Themann den Umgang mit Expert*innendissensen zur Zwischenlagerung in drei exemplarisch betrachteten Foren auf. Diese sind die Endlagerkommission, das NBG und das Forum Zwischenlagerung. Um den Umgang bewerten zu können, werden wesentliche Elemente des Konzeptes der reflexiven Governance und des Diskursmanagements nach Renn (2003) herangezogen. Die Literatur deutet in diesem Zusammenhang darauf hin, dass Expert*innendissense notwendig sind, um reflexive politische Beurteilung zu ermöglichen. Sie wirken stabilisierend, produktiv und legitimierend für politische Entscheidungen. Dafür müssen diese jedoch in der Form institutionalisiert werden, dass ein transparenter und moderierender Umgang mit ihnen möglich wird. Auch müssen die verschiedenen diskursiven Ebenen der Auseinandersetzungen unterschieden werden. Die Analyse der Expert*innendissense verdeutlicht, dass sich in der Bewertung von Risikotechnologien Dissense überlagern und auch die Diskursstränge, die auf Komplexität, Unsicherheit oder Mehrdeutigkeit abzielen, verschwimmen. Darüber hinaus weisen die drei hier betrachteten Foren Schwächen auf hinsichtlich der Transparenz des Einbezugs von Dissensen oder von sich widersprechenden Expert*innen (aus Wissenschaft aber auch aus der Zivilgesellschaft) in Entscheidungsprozesse, der institutionalisierten Absicherung von Gegenexpertise oder der Öffnung für Gegenexpertise generell. Beim NBG deutet sich dagegen eine positive Entwicklung an, die auch dadurch getragen wird, dass das NBG aktiv nach Lernräumen für die im Standortsuchprozess angelegte Bürgerbeteiligung sucht. Für das Forum Zwischenlagerung zeichnet sich jedoch eher eine negative Entwicklung ab, weil die bisherigen Strukturen kaum Raum zur Erprobung des Umgangs mit Dissensen geben (»Zum politischen Umgang mit Expertendissensen «).

Jan Sieveking und Achim Brunnengräber liefern einen Beitrag zur Endlagerdiskursforschung. Sie betrachten den diskursiven Umgang und Gehalt von Begriffen wie Atomkraft oder Kernenergie, Müll, Abfall oder Reststoffe, Oberflächenlager, Zwischenlager oder Endlager sowie weißer und bunter Landkarte. Über solche Begriffe werden auch Expert"innendissense ausgetragen, weshalb der Beitrag den konkurrierenden Bezeichnungen und Bedeutungen dieser Begriffe nachgeht. So kann etwa das Endlager als ewige Gefahr für die Menschheit oder als bestmögliche Lösung angesehen werden. Solche konträren Zuschreibungen oder divides können weder im gesellschaftlichen Diskurs noch von Expert*innen einfach aufgelöst, sprich in einen eindeutigen Sachverhalt oder ein einheitliches Begriffsverständnis überführt werden. Sie sind vielmehr Bestandteil eines Diskursfeldes, auf dem in sozialen Auseinandersetzungen um die Deutungshoheit gerungen wird. Die Begriffe erzählen darüber hinaus viel über das im Endlagerdiskurs implizit Gesagte - aber auch das, was unerwähnt bleibt und jenseits der Grenzen des Diskurses liegt. Die begriffsanalytische Auseinandersetzung mit den genannten Begriffen wird durch eine kritische diskurstheoretische Reflexion verortet und ergänzt (»Der socio-technical divide im Endlagerdiskurs»).

Die vergleichende Analyse von Regulierungs- und Aufsichtsbehörden in Belgien, Kanada und der Bundesrepublik Deutschland von Maria Rosaria Di Nucci, Ana María Isidoro Losada und Erik Laes zeigt, dass die Einbettung in das jeweilige Institutionen- 
gefüge ebenso wie die internen formalen Strukturen und Zuständigkeitsabgrenzungen insbesondere gegenüber der Exekutive und dem Vorhabenträger in hohem Maße von der jeweiligen verfassungsrechtlichen Struktur, dem rechtlich-institutionellen Rahmen sowie der jeweiligen nationalen Regulierungskultur abhängen. Über die Identifizierung und Analyse der zentralen Faktoren und Eigenschaften wird gezeigt, was eine langfristig effektivere und vertrauenswürdige Regulierungs- und Aufsichtsbehörde auszeichnet. Zentrale Aspekte dabei sind die Unabhängigkeit der jeweiligen Regulierungsbehörde sowie die Art des Verhältnisses zur Exekutive, zur Wissenschaft und zur Industrie. Darüber hinaus ist die Rolle des Regulators in der Gestaltung der Öffentlichkeitsbeteiligung sehr unterschiedlich. Das Ergebnis weist auf eine deutliche Herausforderung für das Bundesamt für die Sicherheit der nuklearen Entsorgung (BASE) als Regulierungsbehörde und Träger der Öffentlichkeit hin. Die Tatsache, dass in den untersuchten Ländern andere Instanzen als in der Bundesrepublik für die Öffentlichkeitsbeteiligung verantwortlich sind und Zuständigkeiten anders ausgewiesen wurden, lässt Raum für Überlegungen, ob es sich hierbei um eine Kernaufgabe des Regulators handelt bzw. handeln sollte (»Institutionelle Herausforderungen bei der Endlagerung hochradioaktiver Abfälle«).

\section{Teil III: Reversibilität in Entscheidungsprozessen}

Im dritten Teil wird die Logik reversibel gestalteter Langzeitprozesse analysiert, die auf Neubewertungen von Wissensbeständen und gesellschaftspolitische Entwicklungen flexibel reagieren können. Es wird herausgearbeitet, welche Regeln und Kriterien dafür anwendbar sind und wie diese kommuniziert werden können. Ein Kriterium ist, dass Fehlentscheidungen, die zu unerwünschten Ergebnissen führen könnten, entweder nicht getroffen werden oder aber zumindest revidierbar sein sollten. Dies geschieht vor dem Hintergrund, dass die Komplexität technologischer Infrastrukturen und gesellschaftlicher Anforderungen zugenommen und sich die Streuung und Genese neuer Information und neuen Wissens beschleunigt haben. Insbesondere langfristige Projekte müssen im Stande sein, einen zeitlichen Wandel der Rahmenbedingungen zu berücksichtigen. Aus diesem Grund wird - wie auch im Kontext der Entsorgung hochradioaktiver Abfälle - zunehmend die Reversibilität von Planungs- und Entscheidungsprozessen gefordert. $\mathrm{Zu}$ dem gesetzlich verankerten Anspruch nach Reversibilität im Standortauswahlverfahren (StandAG 2017, \$2) sind darüber hinaus Ansprüche an ein reversibles Entsorgungssystem insgesamt zu berücksichtigen, die auf dem Abschlussbericht der Endlagerkommission (2016) basieren. Ein Konzept dafür, wie reversible Planungs- und Entscheidungsprozesse gewährleistet werden können, gibt es bislang nicht. Der Beitrag »Reversibilität im Kontext der Entsorgung hochradioaktiver Abfälle« von Melanie Mbah, Bettina Brohmann, Saleem Chaudry und Roman Seidl diskutiert den Begriff der Reversibilität zunächst aus der Perspektive unterschiedlicher Disziplinen, um diesen dann in den Entsorgungskontext zu stellen und einen ersten konzeptionellen Ansatz von Reversibilität im Entsorgungssystem herauszuarbeiten.

Durch ein gesetzlich geregeltes Verfahren soll der Standort gefunden werden, der die bestmögliche Sicherheit zur Entsorgung der hochradioaktiven Abfälle der Bundesrepublik Deutschland bietet. Das Standortauswahlverfahren soll lernend, selbsthinter- 
fragend sowie reversibel sein. Das Feld ist von vielfältigem Expert"innenwissen und -handeln geprägt. Änderungen im reversibel gestalteten Verfahrensablauf durch Erkenntnisgewinn oder Neubewertung bestehenden Wissens sind zu erwarten. Dissens zwischen Expert*innen kann in diesem Zusammenhang eine wesentliche Rolle spielen. Um Handlungsempfehlungen für reversible Verfahren im Allgemeinen und das Standortauswahlverfahren im Besonderen zu formulieren, müssen die Mechanismen und Wirkungen von Expert*innendissensen besser verstanden werden. So kann Dissens über den wissenschaftlichen Status-Quo fruchtbar sein, da er Unsicherheiten im Wissen aufdeckt und neue Forschung ermöglicht. Andererseits kann er bei Bürger*innen und Entscheider*innen zu Verunsicherung führen. Expert"innendissens kann darüber hinaus instrumentell eingesetzt werden, um beispielsweise politische Ziele zu verfolgen.

Im Hinblick auf die Reversibilität des Standortauswahlverfahrens soll der Beitrag von Saleem Chaudry und Roman Seidl (»Expertendissens und das reversible Verfahren der Suche nach einem Endlagerstandort für hochradioaktive Abfälle«) helfen zu klären, ob und wie Expert*innendissens auf Entscheidungsprozesse einwirken kann. Dazu wird, basierend auf Sekundärliteratur, eine Definition von Expert"innendissens erarbeitet sowie das Konzept der Reversibilität näher beschrieben. Das im StandAG festgehaltene Reversibilitätsverständnis wird anderen Ansätzen aus verschiedenen Disziplinen gegenübergestellt. Anhand historischer Fälle von Expert*innendissens im Rahmen von Endlagerprojekten wird eine Typologie entwickelt und untersucht, ob und wie diese historischen Dissense Wirkung im jeweiligen Kontext entfaltet haben. Zudem wird geprüft, welche Lehren daraus für das Standortauswahlverfahren gezogen werden können.

Der Gesetzgeber hat sich im StandAG mit der Begriffsbestimmung in $\$ 1$ Abs. 5 S. 1 und nach Maßgabe der $\$ \$ 12 \mathrm{ff}$. für eine ganz bestimmte Ausgestaltung von Reversibilität entschieden - die Festlegung des Standortes wird für das Jahr 2031 angestrebt. Reversibilität wird hier (bisher) primär als Fehlerkorrektur verstanden, nicht als Rücksprung, wie Ulrich Smeddinck in seinem Beitrag »Reversibilität in Entscheidungsprozessen - Warum wir ein lernendes Verfahren brauchen« zeigt. Es ist das Vorrecht der zuständigen Behörde, sich eine eigene Meinung zu bilden und zur Grundlage ihres weiteren Vorgehens zu machen. Das StandAG zielt aber auch auf die Realisierung des Verfahrens im transparenten Dialog mit der Öffentlichkeit. Daraus lässt sich schließen, dass auch die Entscheidungen über reversible Maßnahmen Gegenstand der Öffentlichkeitsbeteiligung sein sollen - und zwar in einer Art und Weise, die im Dialog erfolgt. Auch das StandAG 2017 - und nicht nur das StandAG 2013 - ist auf Fortentwicklung angelegt. Es ist ein Gesetz neuen Typs, das den Anspruch, Bürger*innen als Mitgestalter*innen des Verfahrens einzubeziehen, konsequent einlöst.

\section{Teil IV: Planungs- und Langzeitprozesse}

Im vierten Teil wird auf die Langzeitplanung der Endlagerung eingegangen, deren zentrale Herausforderung darin besteht, über lange Zeiträume hinweg eine hohe Qualität von Entscheidungs- und Monitoringprozessen sowie Handlungsfähigkeit sicherzustel- 
len. Welche Planungsgrundlagen dafür bestehen oder entwickelt werden müssen und wie sie in Expert*innenwissen einzubinden sind, wird vertieft geprüft.

Ähnlich wie der Beitrag zu soziotechnischen Analoga als Erfahrungshintergrund für ein Endlager von Achim Brunnengräber et al. greifen Peter Hocke, Sophie Kuppler und Stefanie Enderle Vergleichsfälle auf, die unter der Perspektive modernen Regierens über lange Zeiträume und das Aufrechterhalten eines spezifischen Sicherheitsniveaus betrachtet werden. Wenn große technische Infrastrukturen - wie ein nukleares Endlager - so gestaltet werden sollen, dass sie sowohl hohe Sicherheitsanforderungen als auch Erwartungen erfüllen, bedarf es einer Sicherheits- und Fehlerkultur, die mögliche zukünftige Entwicklungen antizipiert. Wie die großtechnischen Pannen und Unfälle verlangt eine zukunftsgerichtete long-term Governance schnelles und reflektiertes Reagieren. Sie gewährleistet durch flexibles und gleichzeitig zielorientiertes Handeln auch bei ungünstigen Bedingungen wie partieller Ungewissheiten ein gutes Nachsteuern und die Mobilisierung problemadäquater Ressourcen. Die einschlägige Forschungsliteratur gibt dazu bisher nur eingeschränkt Auskunft. So untersuchen die drei Autor*innen empirisch große technische Infrastrukturen (Talsperren, Verkehrsüberwachung am Beispiel des Schienenverkehrs sowie Climate Engineering), die ein vergleichbar hohes Maß an Sicherheit - ähnlich wie ein Endlager über sehr lange Zeiträume - zu gewährleisten versuchen. Im Vordergrund steht dabei die Aufgabe, Governanceprozesse zu reflektieren, die das Aufdecken von Fehlern begünstigen. Gleichzeitig geht es in dem Beitrag "Robuste Langzeit-Governance und Notwendigkeiten neuer

Navigation « darum, institutionelle Lernprozesse zu identifizieren und deren Übertragbarkeit auf den Fall der Entsorgung hochradioaktiver Abfälle zu prüfen.

Im Beitrag von Melanie Mbah und Bettina Brohmann wird der Aspekt des organisationalen Lernens diskutiert (»Das Lernen in Organisationen«). Transformationen und Verfahren, die über lange Zeiträume - d.h. über Generationen hinweg - andauern, stellen dabei besondere Anforderungen an das institutionelle bzw. organisationale Lernen. Institutionen müssen hier besonders auf veränderte gesellschaftliche Rahmenbedingungen reagieren und neue Informationen und Wissensbestände in Entscheidungsprozessen berücksichtigen. Transformationen weisen spezifische Kennzeichen auf und stellen damit spezifische Managementanforderungen an Institutionen, um anpassungsfähig und flexibel zu bleiben. Hierzu braucht es die Bereitschaft und Offenheit, Neues aufzunehmen, um Kritik und insbesondere Fehler konstruktiv zu bearbeiten. Des Weiteren braucht es Räume und Formate, die ein Lernen auf verschiedenen Ebenen - des Individuums, des Kollektivs (eine Institution) und zwischen Kollektiven (mehreren Institutionen) - begünstigen, und Anstöße zum Lernen geben. Ausgehend von Transformationen und deren Kennzeichen werden im vorliegenden Beitrag spezifische Managementanforderungen abgeleitet und auf transformative Langzeit-Verfahren am Beispiel der Entsorgung hochradioaktiver Abfälle übertragen. Hierbei werden Konzepte des organisationalen Lernens, unter Berücksichtigung von Change Management Ansätzen, und spezifischen Voraussetzungen für das Lernen zwischen Organisationen diskutiert. Das Standortauswahlverfahren und dessen Akteure werden hierbei beispielhaft herangezogen.

Energietechnische Infrastrukturen zeichnen sich durch eine Vielzahl an Standorten und ihre zeitliche Entgrenzung aus. Die in die Infrastruktur eingebundenen techni- 
schen Anlagen verändern ihren jeweiligen Standort nicht nur physisch, sondern beeinflussen auch das Verhältnis der Anwohner*innen zu ihrem Wohnort, die sogenannte Ortsbezogenheit, welche die Betriebsbedingungen einer solchen Anlage beeinflussen können. In zeitlicher Perspektive erfordern diese Infrastrukturen einen sicheren Betrieb über mehrere Jahrzehnte bis Jahrhunderte. Sie durchlaufen in diesem Zeitraum - versetzt an verschiedenen Standorten - die Phasen der Standortauswahl, des Baus und Betriebs sowie des Nachbetriebs. Ein Abbruch der Kontrolle der Anlagen würde zu unerwünschten Effekten für Mensch und Umwelt führen.

In ihrem Beitrag "Raumsensible long-term Governance zur Bewältigung komplexer Langzeitaufgaben « argumentieren Melanie Mbah und Sophie Kuppler, dass diese besonderen Herausforderungen, d.h. die Vielzahl an Standorten in Verbindung mit den langen Zeithorizonten, einen Governance-Ansatz erfordern, der diese Aspekte aufzugreifen vermag. Nur so können etwaige negative Entwicklungen antizipiert und soweit möglich verhindert werden. Der hier vorgeschlagene Ansatz der raumsensiblen longterm Governance unterliegt Lernprozessen, die im Wechselspiel zwischen lokaler Partizipation, welche Ortsbezogenheiten aufgreift, und übergeordneten Zielen, wie der Entsorgung hochradioaktiver Abfälle, entstehen. Diese Prozesse schaffen die Basis für eine auf Langfristigkeit angelegte Governance, was dazu beitragen kann, einen zukünftigen und unerwünschten Kontrollabbruch zu verhindern.

Im Zusammenhang mit Fragen der Sicherheit weist auch der Beitrag »Achtsamkeit und Fehlerkultur als notwendige Sicherheitsleistung « von Oliver Sträter auf eine weitere spezifische Anforderung der Governance hin: die Bedeutung und Bedingung der Implementierung einer Fehlerkultur für den Aufbau einer notwendigen Sicherheitsleistung. Anhand von Beispielen gravierender Schadensereignisse aus der jüngeren Geschichte wird verdeutlicht, wie wichtig der organisationale Kontext des Betreibers und seine Wechselbeziehungen mit dem gesellschaftlichen Umfeld sind. Für eine solche gelungene Wechselbeziehung diskutiert Sträter den Ansatz einer Hochzuverlässigkeitsgemeinschaft.

Abschließende Überlegungen und Ausblicke zu den soziotechnischen Herausforderungen und den gewonnenen Erkenntnissen, die in diesem Band präsentiert wurden, stellen Bettina Brohmann, Peter Hocke, Achim Brunnengräber und Ana María Isidoro Losada vor und zur Diskussion.

\section{Literatur}

BASE (2020): Kompaktwissen zur Endlagersuche. Das letzte Kapitel, Berlin: Bundesamt für die Sicherheit der nuklearen Entsorgung.

https://www.endlagersuche-infoplattform.de/SharedDocs/Downloads/BASE/DE/ broschueren/bfe/journalisten-broschuere.pdf?_blob=publicationFile\&v=8, zuletzt geprüft am 04.09.2020.

BGE (2020): Zwischenbericht Teilgebiete gemäß $\$ 13$ StandAG, Peine: Bundesgesellschaft für Endlagerung. 443 Seiten.

Brunnengräber, Achim (2017): Jahrhundertprojekt Endlagerung. In: GAIA 26/2 (2017), Editorial, 94-95. 
Brunnengräber, Achim/Hocke, Peter (2014): Bewegung Pro-Endlager? Zum soziotechnischen Umgang mit hochradioaktiven Reststoffen. In: Forschungsjournal Soziale Bewegungen 27 (4): 59-70.

Endlagerkommission (2016): Abschlussbericht der Kommission Lagerung hoch radioaktiver Abfallstoffe, K.-Drs. 268. https://www.bundestag.de/resource/blob/434430/bb 37b21b8e1e7e049ace5db6b2f949b2/drs_268-data.pdf, zuletzt geprüft am 11.11.2020

Gibbons, Michael (1999): Science's new social contract with society. In: Nature 402: 81-84.

Grunwald, Armin (2016): Der lange Weg zum Konsens. Abschlussbericht zum Konsens. In: Politische Ökologie (Heft 146): 124-127.

Hocke, Peter (2016): Technik oder Gesellschaft? Atommüll als sozio-technische Herausforderung begreifen. In: Achim Brunnengräber (Hg.): Problemfalle Endlager. Gesellschaftliche Herausforderungen im Umgang mit Atommüll. Baden-Baden: edition sigma in der Nomos Verlagsgesellschaft, 77-96.

Hocke, Peter/Kallenbach-Herbert, Beate (2015): Always the Same Old Story? Nuclear Waste Governance in Germany. In: Brunnengräber, Achim/Di Nucci, Maria Rosaria/Isidoro Losada, Ana María/Mez, Lutz/Schreurs, Miranda A. (Hg.): Nuclear Waste Governance. An International Comparison. Wiesbaden: Springer VS, 177-201.

Jungk, Robert (1977, Erstveröffentlichung): Der Atom-Staat. Vom Fortschritt in die Unmenschlichkeit, München: Kindler.

Kallenbach-Herbert, Beate/Akinsara-Minhans, Anne/Brohmann, Bettina/Kuppler, Sophie/Hocke, Peter/Bechthold, Elske et al. (2018): Spezifizierung der soziotechnischen Herausforderungen. Arbeitsbericht zum Arbeitspaket 1 in SOTEC-radio. Konzepte und Maßnahmen zum Umgang mit den soziotechnischen Herausforderungen bei der Entsorgung radioaktiver Abfälle, Darmstadt/Karlsruhe: Ms. (Version 25.9.18), 93 Seiten.

Kamlage, Jan-Hendrik/Warode, Jan/Mengede, Anna (2019): Chances, Challenges and Choices of Participation in Siting a Nuclear Waste Repository. In: Brunnengräber, Achim/Di Nucci, Maria Rosaria (Hg.): Conflicts, Participation and Acceptability in Nuclear Waste Governance. An International Comparison, Wiesbaden: Springer VS, 91-110.

Renn, Ortwin (2003): Hormesis and risk communication. In: Human $\&$ Experimental Toxicology 22: 3-24. 